\title{
MAXIMAL SUBFIELDS OF AN ALGEBRAICALLY CLOSED FIELD NOT CONTAINING A GIVEN ELEMENT
}

\author{
FRANK QUIGLEY
}

If $K$ is an algebraically closed extension of a field $Q$, and if $\alpha \in K$, $\alpha \notin Q$, then the set $\subseteq$ of all subfields of $K$ not containing $\alpha$ is inductive when partially ordered by inclusion. A maximal element $M$ of $\Im$ is by definition a maximal field without $\alpha$. Such fields are thus characterized by the property that all their proper extensions (in $K$ ) contain $\alpha$.

Theorems 1-3 describe the structure of $K / M$ in detail, and Theorems 4-6, in view of this structure, give existence proofs more precise than that trivially given by Zorn's Lemma. The methods throughout are those of classical Galois theory; Lemma 2 (and possibly Lemma 4) are known, but, lacking suitable references, we give proofs of both.

All fields to be considered are subfields of $K$, and the characteristic of $K$ is $q \geqq 0$.

LEMMA 1. If $M$ is maximal without $\alpha$, then $K$ is an algebraic extension of $M$.

We observe first that $\alpha$ is algebraic over $M$; for if $\alpha^{2} \notin M$, then $M\left(\alpha^{2}\right) \supset M(\alpha) \supset M\left(\alpha^{2}\right)$, so that $\alpha^{2}$ is algebraic over $M$. Let $\bar{M}$ be the algebraic closure of $M$ in $K$. If $\exists \eta \notin \bar{M}$, then $\eta$ is transcendental over $M$, as is every element of $M(\eta)$ not in $M$. Since $M(\eta) \neq M$, it follows that $M(\eta) \supset M(\alpha)$; thus, since $\alpha \notin M$, it follows that $\alpha$ is transcendental over $M$; a contradiction.

THEOREM 1. If $M$ is maximal without $\alpha$, then there exists a prime number $p$ such that $[N: M]$ is a power of $p$ for every finite normal extension $N$ of $M$. Either $M$ is a perfect field, or else $K$ is a purely inseparable extension of $M$. Furthermore $[M(\alpha): M]=p$, and $M(\alpha)$ is a normal extension of $M$. All pth roots of unity lie in $M$, so that there exists $a \notin M(\alpha)$ such that $a^{p} \in M$ and $M(\alpha)=M(a)$, unless $M$ is perfect and $p=q$.

The proof is based on the following

Lemma 2. Let $N$ be a finite normal separable extension of $F$ and assume that $p$ is a prime number such that $p^{r} \mid[N: F]$ but $p^{r+1}\{[N: F]$. Then there exist subfields $L_{i}$ of $N, 0 \leqq i \leqq r$, such that $L_{i} \supset L_{i+1} \supset F$, $\left[L_{i}: L_{i+1}\right]=p, L_{i} / L_{i+1}$ is normal, $L_{0}=N$, and $p \nmid\left[L_{r}: F\right]$.

Under the hypotheses of the lemma, the Galois group of $N / F$ has

Received by the editors July 10, 1961 . 
a Sylow- $p^{r}$-subgroup $\mathfrak{S}$, which has a composition series $\left\{\mathfrak{S}_{i}, 0 \leqq i \leqq r\right\}$ where $\mathfrak{S}_{r}=\mathfrak{E}$. The fixed fields $L_{i}$ of $\mathfrak{S}_{i}, 0 \leqq i \leqq r$, have the required properties.

To prove the theorem, assume that $b^{a} \in M$ but $b \notin M$; then $M(b)$ $\supset M(\alpha)$ and $[M(b): M]=q$, so that $M(\alpha)=M(b), p=q, \alpha^{q} \in M$, and $M(\alpha)$ is a purely inseparable extension of $M$. If $c \notin M$, then some power $c^{q^{t}}$ is separable over $M$. Thus $c^{q t} \in M$, for otherwise $M\left(c^{q^{t}}\right)$ $\supset M(\alpha)$ and so is an inseparable extension of $M$.

Now suppose that $M$ is perfect. Let $p$ be a prime such that $p \mid[M(\alpha): M]$, and let $N$ be a proper finite normal (hence separable) extension of $M$, so that $N \supset M(\alpha)$ and $p \mid[N: M]$. If $p^{r}$ is the highest power of $p$ dividing $[N: M]$, then, by the lemma with $M=F$, $\exists L_{r} \supset M$ with $\left[N: L_{r}\right]=p^{r}$. Thus $L_{r}=M$; for otherwise, $L_{r} \supset M(\alpha)$ and $p \mid\left[L_{r}: M\right]$. Also $\exists L_{r-1}$ such $N \supset L_{r-1} \supset L_{r}$, so that $\left[L_{r-1}: M\right]=p$. Since then $L_{r-1} \supset M(\alpha)$ and since $p \mid[M(\alpha): M]$, it follows that $M(\alpha)=L_{r-1}$, a normal extension of $L_{r}(=M)$ of degree $p$.

If $p \neq q$ and $e$ is a primitive $p$ th root of unity, then $[M(e): M]$ $\leqq p-1$. Therefore $M(e)=M$; and since $M(\alpha)$ is a cyclic extension of $M$ of degree $p, \exists a \in M(\alpha)$ such that $a^{p} \in M$ and $M(\alpha)=M(a)$.

We call the prime number $p$ of Theorem 1 the exponent of the maximal field $M$ without $\alpha$.

Theorem 2. Let $M$ be a maximal field without $\alpha$ of exponent $p$. For each positive integer $r$ there is at most one extension $M_{r}$ of $M$ such that $\left[M_{r}: M\right]=p^{r}$. Such an $M_{r}$ is generated over $M$ by $\left\{\gamma:[M(\gamma): M] \leqq p^{r}\right\}$, and is a normal extension of $M$, cyclic if $M$ is perfect and purely inseparable otherwise. Furthermore every extension $L \neq K$ of $M$ equals some $M_{r}$.

Assume first that $M$ is perfect.

Let $\left[N_{1}: M\right]=\left[N_{2}: M\right]=p^{r}$, and let $N$ be a finite normal extension of $M$ containing $N_{1}$ and $N_{2}$. If $r=1$, then $N_{1}=M(\alpha)=N_{2}$. If $r>1$, then the Galois group $B$ of $N / M$ has order $p^{s}, s>1$. Since $M(\alpha)$ is the only field between $N$ and $M$ of degree $p$, it follows that the $p$-group (B) has only one subgroup of order $p^{8-1}$. Hence $(S)$ is cyclic $[4, p .119]$, and has precisely one subgroup of order $p^{r}, 0 \leqq r \leqq s$. Thus $N$ has only one subextension of degree $p^{r}$ over $M$, and $N_{1}=N_{2}$. Similarly, this unique (if it exists) extension $M_{r}$ of degree $p^{r}$ is a cyclic extension of $M$. Thus if $[M(\gamma): M] \leqq p^{r}$, then $\gamma \in M_{r}$, so that $M_{r}$ is generated over $M$ by $\left\{\gamma \ni:[M(\gamma): M] \leqq p^{r}\right\}$. Finally, let $L \neq K$ be any extension of $M$. Evidently $M_{r} \subset M_{r+1}$, and since $K$ is algebraic over $M$, we have $K=\bigcup M_{r}$. If for every $r, \exists \gamma \in L \ni:[M(\gamma): M] \geqq p^{r}$, then $L \supset M_{r}$, for all $r$, and $L=K$; otherwise $L=M_{r}$, for some $r$. 
Secondly assume that $K / M$ is purely inseparable, so that $p=q$. Evidently $M_{r}=M^{q^{-r}}$, we prove that $M^{q^{-r}}=M\left(\alpha^{q^{1-r}}\right)$, inductively. If $c^{q} \in M$, then either $c \in M$ or $M(c)=M(\alpha)$, and $M_{1}=M(\alpha)$. If $M_{\text {r }}$ $=M\left(\alpha^{q^{1-r}}\right)$ and $c^{q^{r+1}} \in M$, then $c^{q} \in M_{r}$, and so $c \in M_{1}\left(\alpha^{q^{-r}}\right)=M_{r+1}$, Since $K=\bigcup M^{q^{-}}$, each extension $L \neq K$ of $M$ equals an $M_{r}$.

The preceding paragraph also establishes the following theorem in the case that $K / M$ is purely inseparable.

TheOREM 3. Let $M$ be maximal without $\alpha$, and, for each integer $r \geqq 1$, let $M_{r}$ be generated over $M$ by $\left\{\gamma \ni:[M(\gamma): M] \leqq p^{r}\right\}$. Then, either $\left\{M_{r}\right\}$ is a strictly increasing tower of proper subfields of $K$, or else $p=2$, $q=0$, and $M_{1}=M\left(-1^{1 / 2}\right)=K$, so that $M$ is a maximal orderable subfield of $K$. In the case that $M_{1} \neq K$, there exists $b \in M_{1}$ such that $M_{r}$ $=M\left(b^{p^{1-r}}\right)$ for all $r$, unless $M$ is perfect and $p=q$.

For the proof when $M$ is perfect, we need the following

Lemma 3. Let $M$ be maximal without $\alpha$, and of exponent $p \neq q$. If $M(\alpha)=M(a)$ where $a^{p} \in M$, and if $a^{p^{-1}} \in M(a)$, then $M(a)=M(\epsilon)$, where $\epsilon$ is a primitive $p^{2}$-root of unity.

Since, under the hypotheses, $M\left(a^{p^{-1}}\right)=M(a)$, it follows that $a^{p^{-1}}$ is a zero of an irreducible polynomial $f(X) \in M[X]$ of degree $p$. It is also a zero of the polynomial $X^{p^{2}}-a^{p} \in M[X]$, a polynomial whose roots have the form $\epsilon a^{p^{-1}}$ where $\epsilon$ is a $p^{2}$-root of unity. Since $f(X) \mid X^{p^{2}}$ $-a^{p}$, the roots of $f(X)$ have the form $\epsilon_{i} a^{p^{-1}}, 1 \leqq i \leqq p$, where $\epsilon_{i}^{p^{2}}=1$; hence $\epsilon_{1} \cdots \epsilon_{p} a \in M$, because the constant term of $f(X)$ is in $M$. Thus $a=\epsilon b$, where $b \in M$ and $\epsilon^{p^{2}}=1$. It follows that $M(a)=M(\epsilon b)$ $=M(\epsilon)$.

Returning to Theorem 3, we observe by Theorem 2 that unless $[K: M]<\infty$, the tower $\left\{M_{r}\right\}$ is strictly increasing. If $[K: M]<\infty$, then by a theorem of Artin and Schreier [1; or 3, pp. 48-49], $p=2$, $K=M\left(-1^{1 / 2}\right)$ etc. Now assume that $[K: M]=\infty, p \neq q$, and that $M(\alpha)=M(a)$, where $a^{p} \in M$, by Theorem 1. There are two cases.

If $a^{p^{-1}} \in M_{1}(=M(a))$, then $M_{2}=M_{1}\left(a^{p^{-1}}\right)$. Let $\epsilon$ be a primitive $p^{2}$-root of unity; then $[M(\epsilon): M] \leqq p$, since $\epsilon^{p} \in M$ by Theorem 1 . Thus $\epsilon \in M_{1}$. But $M_{1}$ is maximal without $a^{p_{-}^{-1}}$, and it follows that $a^{p^{-2}} \notin M_{2}$; for otherwise, by Lemma $3, M_{2}=M_{1}\left(a^{p^{-1}}\right)=M_{1}(\epsilon)=M_{1}$, which is a contradiction. Continuing inductively, we find that in this case we may take $b=a$.

On the other hand, if $a^{p^{-1}} \in M_{1}$, then, by Lemma 3, $M_{1}=M(a)$ $=M(\epsilon)$. Now $\exists b \in M(\epsilon) \ni: b^{p^{-1}} \notin M(\epsilon)$, and such an element $b$ is not in $M$; for otherwise $\left[M\left(b^{p^{-1}}\right): M(b)\right]=\left[M\left(b^{p^{-1}}\right): M\right]=p$, and $b^{p^{-1}}$ $\in M(\epsilon)$. Thus $M(a) \supset M(b) \neq M$, and $M(\epsilon)=M(b)$. But $M_{1}$ is now maximal without $b^{p^{-1}}$ and $\left(b^{p^{-1}}\right)^{p} \in M_{1}$. If $b^{p^{-1}} \in M_{1}\left(b^{p^{-1}}\right)$, then 
$M_{1}\left(b^{p^{-1}}\right)=M_{1}(\epsilon)=M_{1}$, which is false. Hence $b^{p^{-2}} \notin M_{1}\left(b^{p^{-1}}\right)$, and we continue the argument as in the first case.

In the proofs which follow, the "Theorem of natural irrationality" $[2$, p. 149] is called (TNI), and $Q$ is any subfield of $K$.

TheORem 4. If $\alpha$ is transcendental over $Q$, and if $p$ is a prime number, then there exists a maximal $M \supset Q$ without $\alpha$ of exponent $p$. When $p=q$, both perfect and imperfect $M$ exist.

Let $\{\beta\} \cup\left\{t_{\mu}, \mu \in I\right\}$ be a transcendence basis of $K / Q$, where $\beta=\alpha^{p}$, or where $\beta=\alpha^{q}-\alpha$ and $p=q$. Let $e$ be a primitive $p$ th root of unity, and let $L=Q\left(e, \beta,\left\{t_{\mu}\right\}\right)$. Then $\alpha \notin L$, and $L$ has an extension $M$ which is maximal without $\alpha$. Also $L(\alpha)$ is a normal extension of $L$ of degree $p[1 ;$ or 2, p. 177]. Since $\alpha \notin M$, it follows that $L(\alpha) \cap M$ $\neq L(\alpha)$; thus $L(\alpha) \cap M=L$, and, if $M$ is perfect, by (TNI), $[L(\alpha): L]$ $=[M(\alpha): M]=p$. If $M$ is imperfect, so that $p=q$, then $\alpha^{p} \in M$ but $\alpha \notin M$, and again $[M(\alpha): M]=p$.

TheOREM 5. Let $\alpha$ be algebraic $\{$ separably algebraic $\}$ over $Q$, and let $p$ be a prime. If there exists a maximal $M \supset Q$ without $\alpha$ of exponent $p$, then there exists a finite algebraic extension $F$ of $Q$ such that $F(\alpha)$ is a normal $\{$ normal separable\} extension of $F$ of degree $p$. On the other hand, if there exists any field $F$ such that $F(\alpha) / F$ is normal and $p \mid[F(\alpha): F]$, then there exists a maximal $M$ without $\alpha$ of exponent $p$. If $E$ is the fixed field of the automorphism group of $F(\alpha) / F$, and if $p \mid[F(\alpha): E]$, then a perfect $M$ exists; if $p \mid[E: F]$, then an imperfect $M$ exists.

If $M$ exists and is imperfect, let $F=Q\left(\alpha^{q}\right)$. If $M$ exists and is perfect, let $\mathfrak{M}$ be the Galois group of $M(\alpha) / M$. Let $N$ be generated over $Q$ by $\left\{\alpha^{\sigma}, \sigma \in \mathfrak{M}\right\}$, and let $\mathbb{S}$ be the automorphism group of $N / Q$. If $E$ is the fixed field of $B$, then $B$ is the Galois group of $N / E$, which is a normal separable extension. Now $N \subset M(\alpha)$, and $M(N)=M(\alpha)$, since $\alpha \in N$. If $F=M \cap N$, then, by (TNI), $[N: F]=[M(\alpha): M]=p$. Finally, $N=F(\alpha)$, since $\alpha \notin F$.

For the second part, suppose $\exists F$ such that $F(\alpha) / F$ is normal and $p^{r} \mid[F(\alpha): E]$ but $p^{r+1}\{[F(\alpha): E]$, where $E$ is the fixed field of the automorphism group of $F(\alpha) / F$. If $E \neq F(\alpha)$, then, by Lemma 2, there exists $L_{1}$ such that $F(\alpha) \supset L_{1} \supset E$ and $\left[F(\alpha): L_{1}\right]=p$, normal. Since $\alpha \notin L_{1}$, there exists a maximal $M$ without $\alpha$ containing $L_{1}$. But then $M \cap F(\alpha)=L_{1}, M(F(\alpha))=M(\alpha)$, and $p=\left[F(\alpha): L_{1}\right]=[M(\alpha): M]$. If $p=q$ and $E \neq F$, then $\alpha$ is inseparable over $F$ and $\left[F(\alpha): F\left(\alpha^{q}\right)\right]=q$. Imbed $F\left(\alpha^{q}\right)$ in a maximal $M$ without $\alpha$; then $[M(\alpha): M]=q$, since $\alpha^{q} \in M$ but $\alpha \notin M$.

THEOREM 6. If $\alpha$ is algebraic over $Q$ and $p$ is a prime, let $N$ be the 
least normal extension of $Q$ containing $\alpha$. Then there exists a maximal $M \supset Q$ without $\alpha$ of exponent $p$ if and only if $p \mid[N: Q]$.

This follows at once from Theorem 5 and the following

LEмма 4. Let $\alpha$ be algebraic over $Q$, and let $N$ be the least normal extension of $Q$ containing $\alpha$. If $p$ is a prime, then there exists a field $F \supset Q$ such that $F(\alpha) / F$ is a normal extension of degree $p$ if and only if $p \mid[N: Q]$.

If $\exists F \ni: F(\alpha) / F$ is normal and separable of degree $p$, then, by (TNI), the Galois groups of $N / N \cap F$ and $N(F) / F$ are naturally isomorphic, and $N(F) \supset F(\alpha) \supset F$. Let $\mathfrak{S}$ comprise all automorphisms of $N(F)$ leaving $F(\alpha)$ fixed, and let $\mathfrak{S}^{\prime}$ be the restriction to $N$ of the automorphisms in $\mathfrak{S}$. The fixed field of $\mathfrak{S}^{\prime}$ is $(N \cap F)(\alpha)$, and $[(N \cap F)(\alpha): N \cap F]=[F(\alpha): F]=p$. Thus $p \mid[N: Q]$. If $F(\alpha) / F$ is inseparable, then $p=q$ and $\alpha^{q} \in F$. Since $\alpha \notin F$, it follows that $\alpha \notin Q\left(\alpha^{q}\right) \subset F$; thus $\left[Q(\alpha): Q\left(\alpha^{q}\right)\right]=q$ and $q \mid[N: Q]$.

Conversely, assume that $p \mid[N: Q]$, and let $\&$ be the automorphism group of $N / Q$. Let $E$ be the fixed field of $(s$. If $p \nmid[N: E]$, then $p \mid[E: Q], p=q$, and we may take $F=Q\left(\alpha^{q}\right)$. If $p \mid[N: E]$, let $\mathfrak{S}_{1}, \cdots, \mathfrak{S}_{8}$ be all the Sylow $p$-subgroups of $\mathfrak{G}$, and let $\mathfrak{E}$ be the group which they generate. Since the $\mathfrak{S}_{i}$ are a complete set of conjugate subgroups of any one of them, $\mathfrak{S}$ is a normal subgroup of $\mathbb{H}$. Let $F_{0}$ be the fixed field of $\mathfrak{W}$, and $F_{i}$ the fixed field of $\mathfrak{S}_{i}$; then $F_{0}=\bigcap_{1}^{s} F_{i}$. We observe that $\alpha \notin F_{0}$. For otherwise, since $F_{0} / E$ is normal and $N \supset F_{0}$, it follows that $F_{0}=N$, and $\mathfrak{S}$ contains only the identity, which is false. Thus $\alpha \notin F_{i_{0}}$ for some $i_{0} \geqq 1$. If $p^{r}$ is the order of $\mathfrak{S}_{i_{0}}$, then $p^{r+1}$ does not divide the order of $\mathbb{G}$, since $\mathfrak{S}_{i_{0}}$ is a Sylow $p$-group, so that $p^{r+1} \nmid[N: E]$ and $\left[N: F_{i_{0}}\right]=p^{r}$. Hence $\left[F_{i_{0}}(\alpha): F_{i_{0}}\right]$ $=p^{t}, 1 \leqq t \leqq r$. If $G$ is the subgroup of $\&$ leaving $F_{i_{0}}(\alpha)$ fixed, then $G\left(\subset \mathfrak{S}_{i_{0}}\right)$ is a $p$-group, so $\exists G^{\prime} \ni: G \subset G^{\prime} \subset \mathfrak{W}_{i_{0}},\left(G^{\prime}: G\right)=p$, and $G$ is a normal subgroup of $G^{\prime}$. Let $F$ be the fixed field of $G^{\prime}$; then $\left[F_{i_{0}}(\alpha): F\right]$ $=\left(G^{\prime}: G\right)=p$, and $F_{i_{0}}(\alpha) / F$ is normal. Finally $F_{i_{0}}(\alpha) \supset F(\alpha)$, but $F(\alpha)$ $\neq F$, since $\alpha \notin F$; thus $F_{i_{0}}(\alpha)=F(\alpha)$.

\section{REFERENCES}

1. E. Artin and O. Schreier, Eine Kennzeichnung der reell abgeschlossenen Körper, Abh. Math. Sem. Univ. Hamburg 5 (1927), 225-231. 1950.

2. N. Bourbaki, Algèbre, Ch. 5, Actualités Sci. Ind., no. 1102, Hermann, Paris,

3. - Algèbre, Ch. 6, Actualités Sci. Ind., no. 1179, Hermann, Paris, 1952.

4. H. Zassenhaus, The theory of groups, Chelsea, New York, 1949.

TUlane UNIVERSITY 